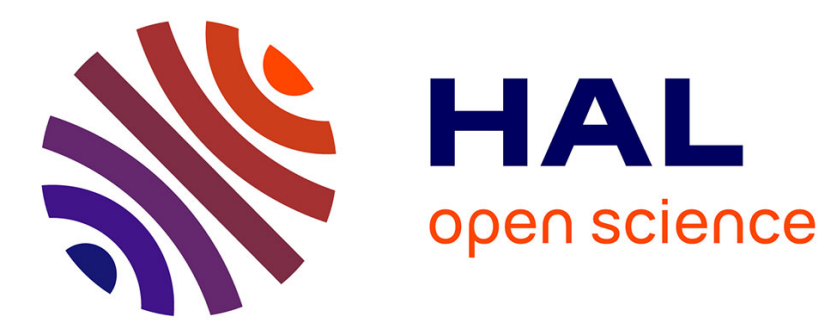

\title{
Utilisation en microscopie électronique des pertes d'énergie des électrons pour l'analyse chimique locale \\ B. Jouffrey, Jean Sévely
}

\section{To cite this version:}

B. Jouffrey, Jean Sévely. Utilisation en microscopie électronique des pertes d'énergie des électrons pour l'analyse chimique locale. Revue de Physique Appliquée, 1976, 11 (1), pp.101-111. 10.1051/rphysap:01976001101010100 . jpa-00243949

\section{HAL Id: jpa-00243949 https://hal.science/jpa-00243949}

Submitted on 1 Jan 1976

HAL is a multi-disciplinary open access archive for the deposit and dissemination of scientific research documents, whether they are published or not. The documents may come from teaching and research institutions in France or abroad, or from public or private research centers.
L'archive ouverte pluridisciplinaire HAL, est destinée au dépôt et à la diffusion de documents scientifiques de niveau recherche, publiés ou non, émanant des établissements d'enseignement et de recherche français ou étrangers, des laboratoires publics ou privés. 


\title{
UTILISATION EN MICROSCOPIE ÉLECTRONIQUE DES PERTES D'ÉNERGIE DES ÉLECTRONS POUR L'ANALYSE CHIMIQUE LOCALE
}

\author{
B. JOUFFREY et J. SEVELY \\ Laboratoire d'Optique Electronique du C. N. R. S., \\ 29, rue J.-Marvig, B. P. 4347, 31055-Toulouse Cedex, France
}

\begin{abstract}
Résumé. - L'analyse des pertes d'énergie des électrons ayant traversé une couche mince permet de mettre en évidence l'excitation de plasmons et d'électrons des niveaux atomiques profonds. L'étude des plasmons donne des informations sur la densité des électrons de la bande de valence. Ils peuvent en particulier être utilisés dans le cas d'alliages dont la composition moyenne est connue pour déterminer la répartition des divers éléments.

L'étude de l'excitation des électrons des niveaux atomiques profonds permet d'envisager une méthode d'analyse chimique à l'échelle de la microscopie électronique. La précision de cette méthode atteint à l'heure actuelle une valeur de $5 \times 10^{-3}$. Quelques exemples d'applications sont donnés dans le cas particulier d'échantillons lunaires.
\end{abstract}

\begin{abstract}
Energy loss analysis in the electron microscope is useful for studying the electronic structure of thin films : the free electron densities in the valence bands are related to the plasmon losses. In particular, the distribution of known types of impurities can be determined in alloys.

Deep inner shell excitation yields a method of chemical microanalysis : the sensitivity is now about $5 \times 10^{-3}$. Applications for moon dust particles will be shown.
\end{abstract}

1. Introduction. - Lorsqu'il traverse l'objet, à la suite de son interaction avec les divers éléments de celui-ci, l'électron a une très forte probabilité de perdre une partie de son énergie. Il garde ainsi en mémoire les divers événements qu'il a subis au cours de son passage dans l'objet. Ruthemann [1] avait le premier montré que cette information se traduit par des pertes d'énergie discrètes, caractéristiques du type d'atomes rencontrés. La microscopie électronique avec analyse et sélection d'énergie des électrons permet d'obtenir une analyse de la composition chimique locale d'une très petite portion de l'objet. Celle-ci peut théoriquement être de l'ordre de grandeur du pouvoir de résolution du microscope. Bien que proposée de longue date par Hillier et Baker [2], cette méthode d'analyse des objets s'est surtout développée à partir de 1962 avec les travaux de l'équipe de Castaing [3].

Les divers processus de diffusion qu'un électron peut subir à la traversée d'un objet, se répartissent en trois groupes auxquels correspondent des modifications d'énergie différentes.

1.1 LeS PROCESSUS ÉlASTIQUES. - Diffusion élastique en milieu amorphe ou cristallin.

1.2 LES PROCESSUS DUS A LA DIFFUSION THERMIQUE PAR INTERACTION ÉLECTRON-PHONON. - L'angle de diffusion peut être très grand, mais la modification d'énergie, liée à la création ou à l'annihilation de phonons est très faible, de l'ordre de $1 / 100 \mathrm{~d}$ 'eV. Avec des systèmes dispersifs dont le pouvoir de résolution en énergie est généralement de l'ordre de $1 \mathrm{eV}$, ils ne peu- vent être directement séparés des processus de diffusion élastiques d'où leur nom de processus quasi élastiques.

\subsection{LES PROCESSUS DE DIFFUSION PROPREMENT INÉ-} LASTIQUES. - Ils peuvent provenir :

De l'excitation d'oscillations collectives du nuage électronique, connues sous le nom d'oscillations de plasma ou plasmons. Elles entraînent des pertes d'énergie plus ou moins discrètes, variant de quelques électron-volts à quelques dizaines d'électron-volts.

De l'excitation d'électrons individuels. Elle conduit à des pertes d'énergie dont la valeur s'étend de quelques électron-volts pour les électrons des couches externes à plusieurs kiloélectron-volts pour les électrons des couches profondes d'éléments de numéro atomique élevé.

De la diffusion due au rayonnement de freinage et à l'effet Cerenkov.

En introduisant un système dispersif dans la colonne optique du microscope, il est possible de séparer les électrons correspondant à une perte d'énergie donnée dans une portion choisie de l'image [4] et éventuellement d'isoler certains de ces électrons pour former l'image en ne conservant que les électrons appartenant à une bande d'énergie déterminée. On peut concevoir que la recherche des électrons correspondant à une perte d'énergie caractéristique dans un très petit volume de l'objet, conduise à une analyse très localisée des solides. 
2. Les dispositifs de mise en évidence et de mesure des pertes d'énergie. - Les divers types de pertes d'énergie ont été étudiés en microscopie électronique avec analyse d'énergie entre $20 \mathrm{kV}$ environ et $1200 \mathrm{kV}$. Les dispositifs adaptés sur les microscopes utilisent les propriétés dispersives des champs électrostatiques et magnétiques. Klemperer [5] et Metherell [6] ont donné une bibliographie de ces divers dispositifs. Elle vient d'être complétée par Colliex [7]. Pour notre part, nous utilisons à basse tension, c'est-à-dire à $60 \mathrm{kV}$, un système de filtrage d'énergie du type Castaing-Henry [2] adapté sur un microscope commercial Siemens Elmiskop I [8]. La résolution en énergie obtenue est de $\pm 1 \mathrm{eV}$. A plus haute tension, c'est-à-dire, entre 300 et $1200 \mathrm{kV}$, les pertes d'énergie sont analysées à l'aide d'un prisme magnétique placé après l'écran d'observation du microscope [9]. Ce dispositif a l'avantage important de ne pas perturber le fonctionnement du microscope. Il permet d'analyser les pertes d'énergie dans une aire sélectionnée de l'objet inférieure à une fraction de micron. Son pouvoir de résolution en énergie est de $\pm 1,5 \mathrm{eV}$ à $1000 \mathrm{kV}$.

La figure 1 donne un exemple de spectre des pertes d'énergie d'électrons de $1 \mathrm{MeV}$ obtenu avec un échantillon d'aluminium de $2000 \AA$ d'épaisseur. Sur le spectre, on distingue d'abord une première raie. Elle correspond aux électrons diffusés élastiquement ou quasi-élastiquement. Ensuite vient un ensemble de raies équidistantes, séparées par $15 \mathrm{eV}$ environ. Elles sont associées aux pertes par excitation de plasmons. Au-delà d'un seuil situé à $1540 \mathrm{eV}$, apparaît une distribution associée à l'excitation du niveau atomique $\mathrm{K}$ de l'aluminium. Ces distributions caractéristiques se superposent à un fond continu dont l'intensité augmente avec l'épaisseur de l'échantillon qui est en général attribué à l'excitation d'électrons individuels.

Comme il apparaît sur la figure 1, deux types de pertes caractéristiques peuvent être mis en évidence : celles liées à l'excitation des plasmons et celles produites par l'excitation de niveaux atomiques profonds.

3. Plasmons. - Dans un échantillon dont l'épaisseur est telle qu'il peut être observé en microscopie électronique, le processus essentiel de pertes d'énergie est l'excitation de plasmons. Ce sont les développements théoriques de Bohm, Pines et Nozières [10] qui ont montré l'origine collective de ce type de pertes. Le faisceau d'électrons excite le gaz des électrons libres et celui-ci exécute des oscillations longitudinales analogues à des oscillations de plasma. La quantité d'énergie transférée à l'ensemble des électrons libres apparaît sous forme de multiples entiers d'un quantum élémentaire d'énergie $\hbar \omega_{\mathrm{p}}$ où $\omega_{\mathrm{p}}$ est la pulsation du plasma et

$$
\begin{gathered}
\hbar=\frac{h}{2 \pi} \quad(h=\text { cte de Plank }) \\
\omega_{\mathrm{p}}^{2}=\frac{4 \pi n e^{2}}{m}
\end{gathered}
$$
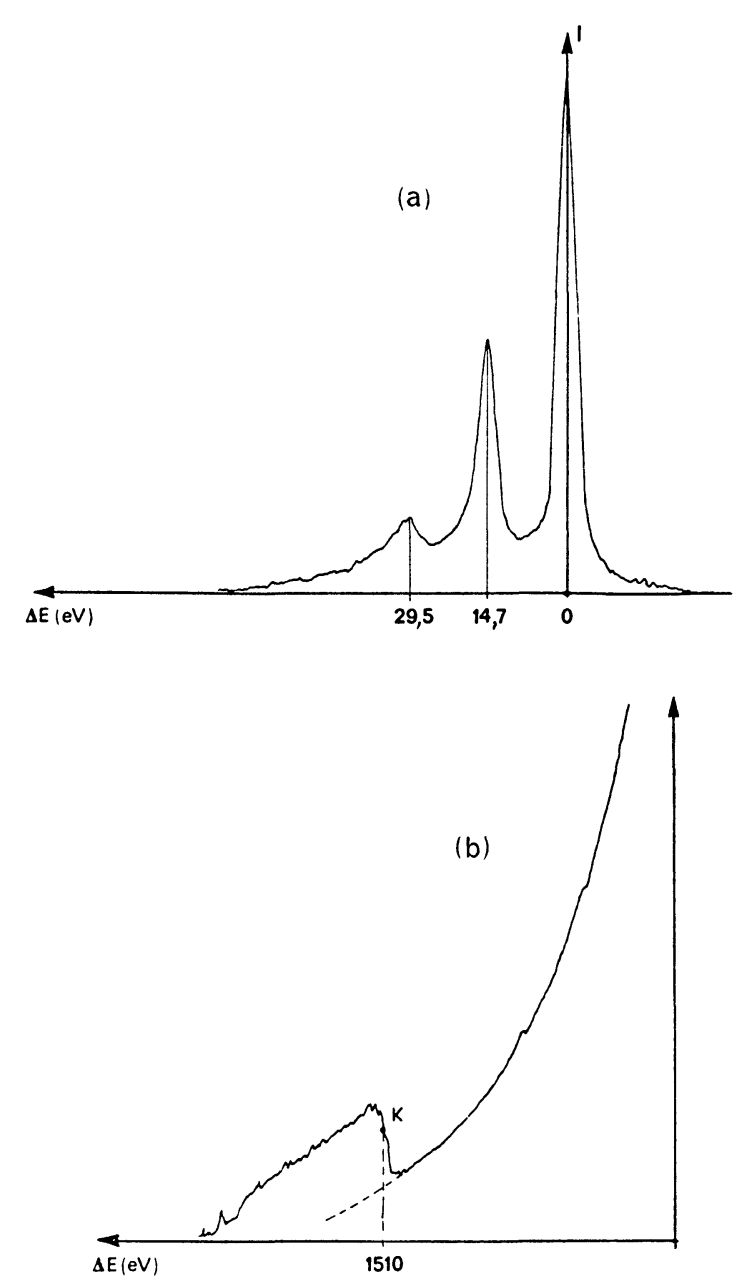

FIG. 1. - Spectre de pertes d'énergie d'électrons de $1 \mathrm{MeV}$ dans un échantillon d'aluminium d'épaisseur $2000 \AA$. a) Pertes d'énergie inférieures à $50 \mathrm{eV}$. $b$ ) Pertes d'énergie au voisinage du seuil d'excitation $\mathrm{K}$. L'échelle des abscisses, $\Delta E$, est multipliée par 0,25 . L'échelle des ordonnées, $I$, est multipliée par 300 .

$n$ est la densité des électrons libres ( 3 par atome pour l'aluminium), $m$ est la masse au repos de l'électron et $e$ sa charge.

La longueur d'onde des oscillations de plasmons a une limite inférieure de l'ordre de la distance moyenne entre les électrons du plasma. Ceci entraîne que le nombre d'onde d'oscillations a une valeur maximale $k_{\mathrm{c}}$ et l'angle de diffusion une valeur maximale $\theta_{c}$, appelée angle de coupure [11].

$$
\theta_{\mathrm{c}}=\frac{k_{\mathrm{c}}}{k}
$$

au-delà de $\theta_{\mathfrak{c}}$, la diffusion par plasmon est négligeable. Pour l'aluminium à $60 \mathrm{kV}$

$$
\theta_{\mathrm{c}} \sim 10^{-2} \text { radian }
$$

à $1000 \mathrm{kV}$

$$
\theta_{\mathrm{c}} \sim 2 \times 10^{-3} \text { radian } .
$$

Ceci montre que tous les électrons diffusés par plasmons peuvent être collectés dans un microscope et 
qu'ils jouent un rôle important dans les problèmes de contraste.

Le libre parcours moyen $\lambda_{\mathrm{p}}$ associé à ce processus est :

$$
\lambda_{\mathrm{p}}=\frac{\hbar \omega_{\mathrm{p}}}{\mathrm{d} E / \mathrm{d} x}
$$

où $\mathrm{d} E / \mathrm{d} x$ est la perte d'énergie correspondante par unité de longueur de la trajectoire de l'électron incident. La probabilité pour un électron incident d'exciter $n$ plasmons à la traversée d'un échantillon d'épaisseur $x$, obéit à la loi de Poisson. Comme il apparaît sur la figure 2, ceci se traduit par une augmentation de l'intensité et du nombre des pics de plasmons avec $x$.
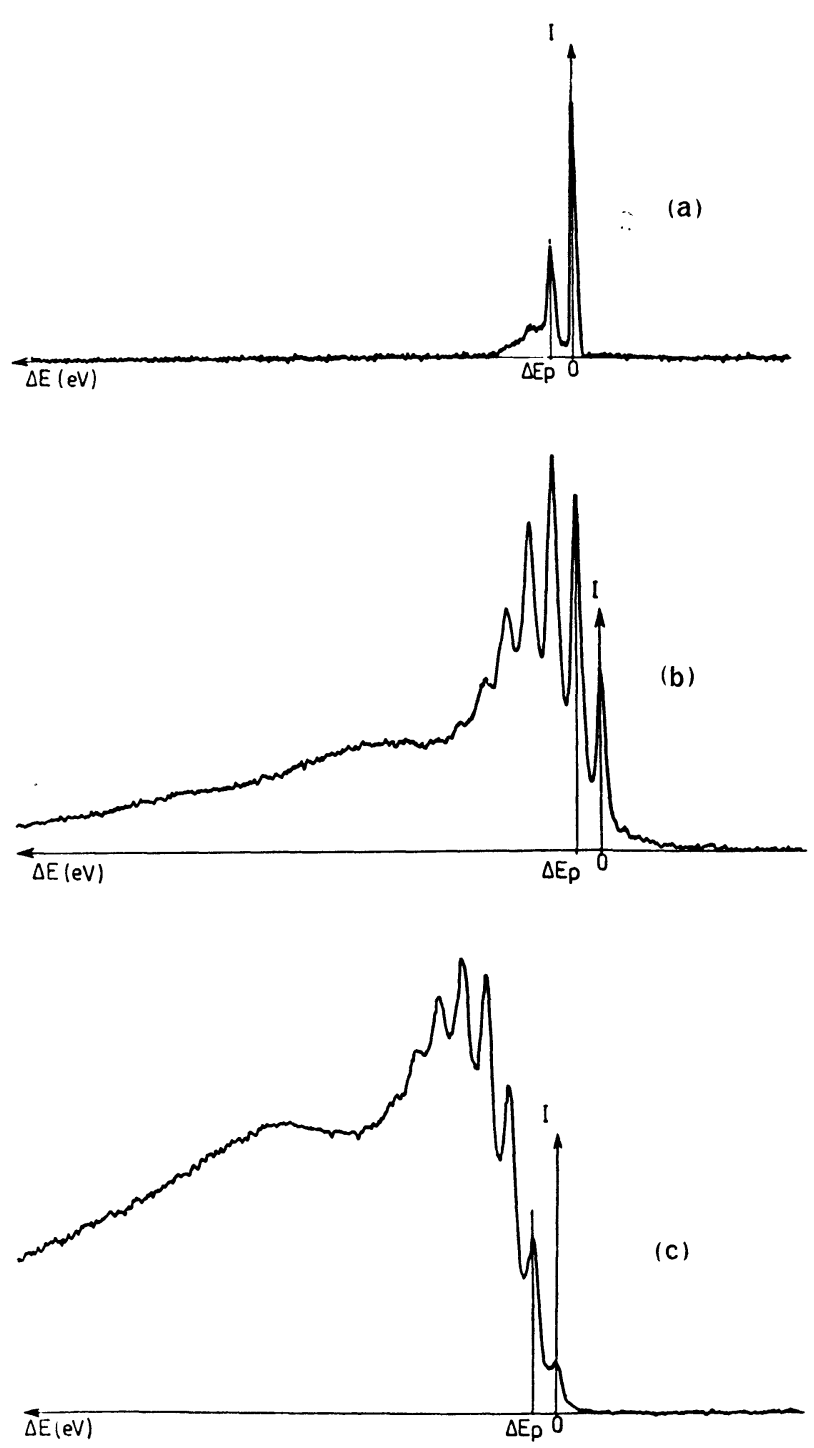

FIG. 2. - Spectre de pertes d'énergie d'électrons de $1 \mathrm{MeV}$ dans des échantillons d'aluminium d'épaisseur croissante.

$$
\text { a) } x=980 \AA, b) x=7600 \AA, c) x=1,018 \mu \mathrm{m} \text {. }
$$

Les valeurs du libre parcours moyen correspondant à l'excitation de plasmons dans le cas du carbone et de l'aluminium ont été déterminées entre 60 et $1200 \mathrm{kV}$ [12]. Les résultats sont rassemblés dans la figure 3. A $60 \mathrm{kV}$, les valeurs mesurées par Y. Kihn [8] sont $560 \pm 85 \AA$ pour le carbone et $960 \pm 130 \AA$ pour l'aluminium. Elles sont proches des valeurs mesurées à la même tension par Burge et Misell [13] et données

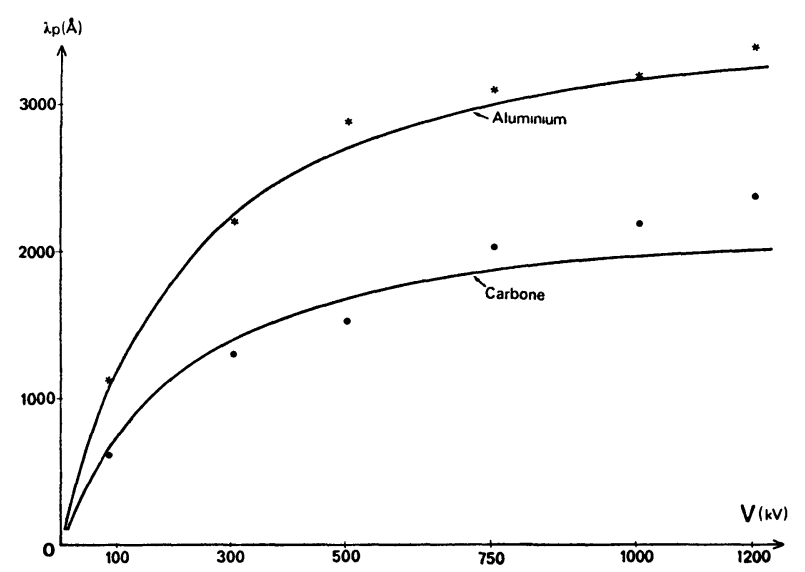

Fig. 3. - Variation du libre parcours moyen des plasmons, $\lambda_{p}$, dans l'aluminium et le carbone en fonction de la tension accélératrice des électrons incidents $*$ et $\bullet$ représentent les valeurs expérimentales. La courbe donne la variation de la valeur de $\lambda_{\mathrm{p}}$, calculée en utilisant la formule d'Ashley et Ritchie :

$$
\frac{1}{\lambda_{\mathrm{p}}^{\mathrm{AR}}}=\frac{e^{2} \omega_{\mathrm{p}}}{2 \hbar C^{2} \beta^{2}}\left[\log \left(\frac{C \cdot k_{\mathrm{c}}}{\omega_{\mathrm{p}}}\right)^{2}+\log \beta^{2}\right] .
$$

dans le tableau I. Les résultats sont rassemblés dans la figure 3. Ils sont en bon accord avec les valeurs calculées en utilisant la formule de Ashley et Ritchie [14] et ils confirment la validité du traitement de Pines [15], dans le cas relativiste, pour rendre compte de l'interaction électron-plasmon.

\section{TABLEAU I}

$\begin{array}{lllllll}\begin{array}{l}\text { Energie des électrons } \\ \text { incidents }(\mathrm{keV})\end{array} & 23 & 30 & 40 & 50 & 60 & 70 \\$\cline { 2 - 6 } & - & - & - & - & - & - \\ $\left.\lambda_{\mathrm{p}}(\mathrm{en} \AA) & 380 & 470 & 490 & 695 & 815 & 920 \\ \text { Valeur expérimentale } & 388 & 493 & 624 & 747 & 863 & 973\end{array}\right\} \mathrm{Al}$

$\lambda_{\mathrm{p}}$ augmente lorsque l'énergie des électrons incidents $s$ 'élève et tend vers une valeur limite aux plus hautes énergies. Ceci entraîne que la probabilité d'observer des pertes d'énergie par plasmon diminue au fur et à mesure que l'énergie des électrons incidents s'élève et va dans le sens de l'intérêt d'utiliser les très hautes tensions en microscopie électronique pour réduire les effets de l'aberration chromatique sur la limite de résolution.

Application des plasmons a L'ANAlyse ChimiQUE. - Si les pertes d'énergie par plasmon se traduisent par des raies suffisamment intenses et bien localisées dans le spectre des pertes d'énergie, l'information que l'on peut en extraire est faible. Elle est relative à la densité des électrons libres. Aussi n'ont-elles été utilisées que dans des cas simples comme celui de solutions 
solides binaires. En mesurant les variations de $\Delta E_{\mathrm{p}}$, Cundy, Metherell et Whelan [16] ont mis en évidence les variations de concentration en magnésium qui apparaissent au voisinage des joints de grain dans un alliage aluminium-magnésium. La même méthode a été ensuite appliquée à d'autres types d'alliages d'alumiminium par Doig, Edington et Jacobs [17] et par Spalding, Villagrana et Chadwick [18]. Cette méthode impose d'avoir une information préalable sur la composition de l'objet étudié.

Les pertes par plasmon ont également été utilisées par Castaing R., Henry L. et El Hili [19] pour déterminer la structure locale d'échantillons métalliques à l'aide d'images filtrées. Le filtre d'énergie est réglé pour ne laisser passer que les électrons dont la perte d'énergie par plasmon est caractéristique d'un élément donné dans le composé : l'image obtenue présente la distribution du composé dans la région examinée.

La figure 4 montre deux images obtenues en filtrant les électrons ayant subi une perte d'énergie $\Delta E$ de
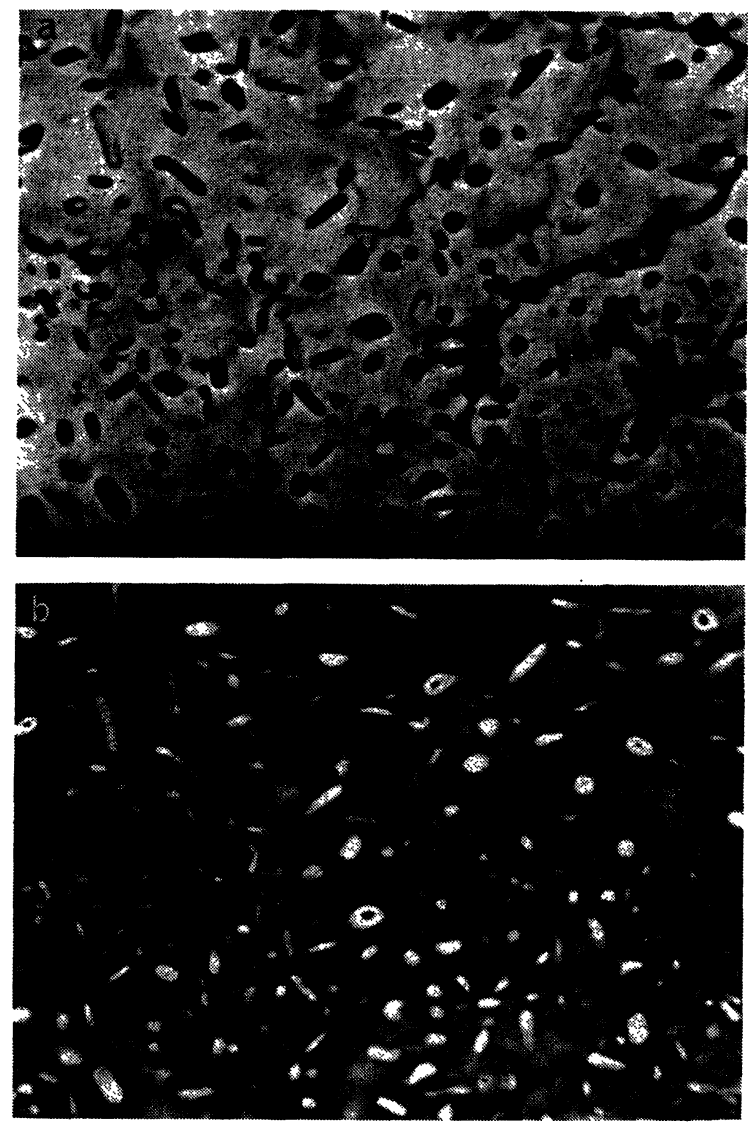

Fig. 4. - Alliage Al-Mg 2,6 \%-Zn 7,6\% aminci électrolytiquement (cliché El Hili). a) Image filtrée $\Delta E \sim 15 \pm 1 \mathrm{eV}$. b) Image filtrée $\Delta E \sim 22 \pm 1 \mathrm{eV}$.

$15 \pm 1 \mathrm{eV}$ caractéristique de la matrice d'aluminium et de $22,5 \pm 2,5 \mathrm{eV}$ caractéristique du précipité $\mathrm{MgZn}_{2}$.

La figure 5 due à Duval et Henry $\left({ }^{1}\right)$ montre trois

(1) Duval, Ph., Henry, L. : communication privée.
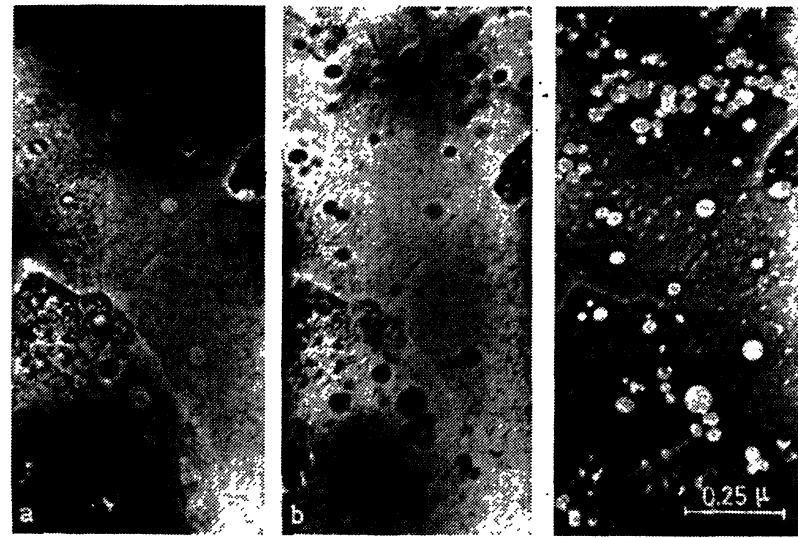

Fig. 5. - Alliage $\mathrm{Al}-\mathrm{Be}$, précipités de béryllium. a) Image filtrée sans perte. b) Image filtrée $\Delta E \sim 15 \mathrm{eV}$ (aluminium). c) Image filtrée $\Delta E \sim 20 \mathrm{eV}$ (béryllium).

images de la même région d'un alliage d'aluminium contenant des précipités de béryllium dont les pertes par plasmons sont respectivement 15 et $20 \mathrm{eV}$. Sur les clichés, chaque phase apparaît en clair sur l'image formée à partir de l'une de ces pertes caractéristiques. Mais cette identification des diverses phases d'un précipité est compliquée par le fait que l'énergie des plasmons est modifiée lorsque le diamètre des précipités est inférieur à 100 Å. Ceci a été utilisé par Henoc et Henry [20] pour identifier de très petites bulles d'hélium au sein d'échantillons métalliques (Fig. 6).
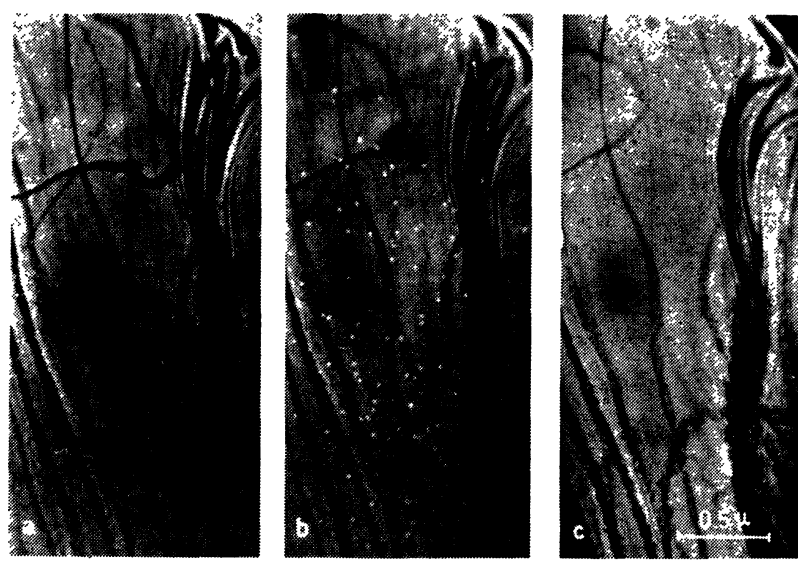

Fig. 6. - Bulles d'hélium dans un échantillon d'aluminiumlithium recuit après irradiation : $a$ ) Image élastique. $b$ ) Perte $\Delta E \sim 11 \mathrm{eV}$. c) Perte $\Delta E \sim 15 \mathrm{eV}$. Sur les images a) et $c$ ) correspondant à une perte nulle ou à la perte $15 \mathrm{eV}$ de la matrice à l'état massif, les bulles sont pratiquement invisibles. Elles apparaissent en clair sur l'image $b$ ) correspondant à la perte $11 \mathrm{eV}$, valeur de l'énergie de plasmon à la surface d'une bulle.

Malgré ces quelques exemples d'applications des pertes par plasmons pour l'analyse de la matière, il semble que cette méthode ne puisse être que d'un emploi très limité. Ceci est surtout dû à la faible importance des variations des pertes d'énergie de ce type d'un élément à l'autre : quelques dizièmes d'eV dans une gamme très limitée comprise entre 10 et $25 \mathrm{eV}$. 
Il semble que ces difficultés pourront être évitées en utilisant les pertes d'énergie beaucoup plus importantes produites par l'excitation de niveaux atomiques.

4. Excitation de niveaux atomiques profonds. L'électron incident peut avoir une interaction de caractère individuel avec les électrons de l'atome. Celle-ci donne lieu à une perte d'énergie caractéristique quand elle se produit avec des électrons appartenant à une couche atomique. Sous l'impact de l'électron incident, l'électron de l'atome subit une transition vers un état inoccupé d'énergie plus élevée, au-delà du niveau de Fermi. Ceci se traduit dans le spectre des pertes d'énergie par l'apparition au-delà d'un certain seuil, d'une structure étendue qui se superpose au fond continu. Les pertes d'énergie correspondantes à ce seuil varient, suivant la couche atomique et le numéro atomique de l'élément, de quelques $\mathrm{eV}$ à plusieurs $\mathrm{keV}$.

Les spectres de la figure 7 donnent la distribution des pertes d'énergie observées à $60 \mathrm{kV}$ dans des échan-

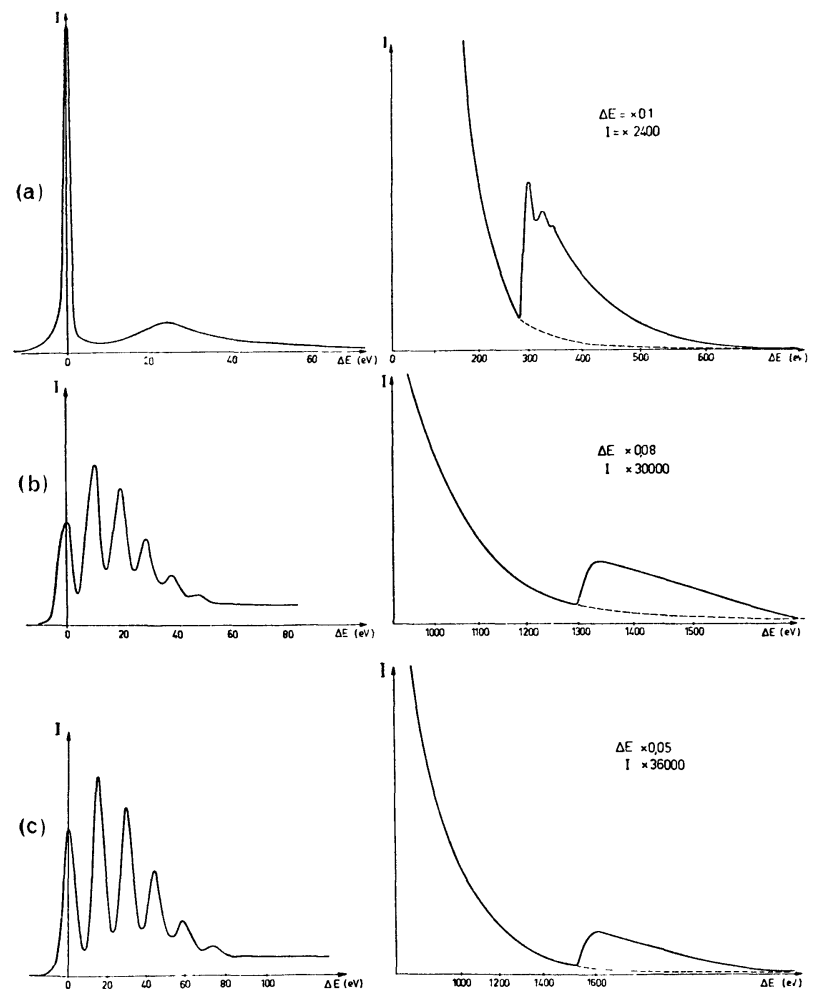

Fig. 7. - Pertes d'énergie observées à $E_{0}=60 \mathrm{keV}$. a) Carbone amorphe $x=500 \AA$. b) Magnésium poli électrolytiquement. c) Aluminium évaporé $x=1000 \AA$.

tillons minces de carbone, de magnésium et d'aluminium. Les seuils d'excitation des niveaux $\mathrm{K}$ apparaissent respectivement à 285,1310 et $1540 \pm 10 \mathrm{eV}$ [8]. Des excitations de niveaux $K$ dans des éléments du numéro atomique plus élevé ont été mises en évidence avec l'analyseur du microscope 1,2 MV [21]. La figure 8 donne un exemple des spectres de pertes d'énergie du titane et du nickel obtenus à $1000 \mathrm{kV}$. Les

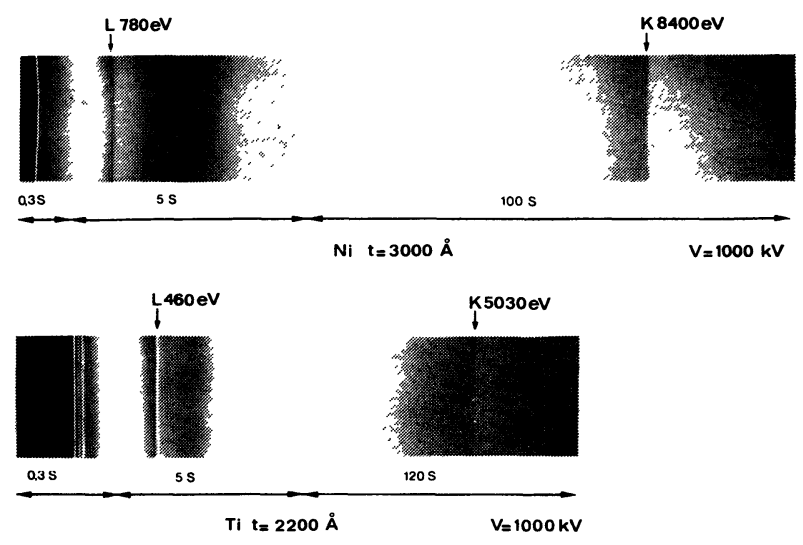

Fig. 8. - Spectres de pertes d'énergie obtenues à $1000 \mathrm{keV}$ dans un échantillon de nickel d'épaisseur égale à $3000 \AA$ et de titane d'épaisseur égale à $2200 \AA$. Les diverses régions de chaque spectre sont obtenues avec des temps de pose différents.

pertes caractéristiques dues à l'excitation du niveau $\mathrm{L}_{23}$ apparaissent respectivement à 460 et $780 \mathrm{eV}$ et celles dues à l'excitation du niveau $\mathrm{K}$ à 5030 et $8400 \mathrm{eV}$. Dans les spectres, les distributions sont bien séparées d'un élément à l'autre et leur seuil est situé à des valeurs très proches des énergies de liaison des électrons excités Elles sont caractéristiques de chaque élément et il est tentant de les utiliser pour une analyse chimique locale. Leur étude est compliquée par le fait qu'elles sont peu intenses et qu'elles sont superposées à un fond continu qui peut être très important; par ailleurs, les effets de diffusion multiple modifient la forme de la distribution. Leur mise en évidence impose des temps d'exposition relativement longs, plusieurs centaines de fois supérieurs aux temps d'exposition relatifs aux plasmons.

4.1 Règles DE TRANSition. - En dépit de la faiblesse de l'intensité correspondant à ces excitations et de la difficulté de les interpréter directement, les premiers spectres relatifs aux transitions $\mathrm{K}$ ou $\mathrm{L}$ ont permis

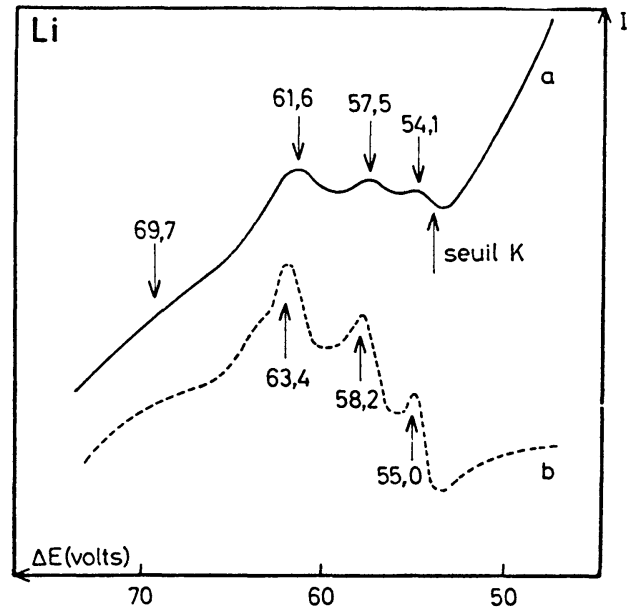

Fig. 9. - Spectre K du lithium. $a$ : Spectre obtenu par Colliex et Jouffrey. $b$ : Spectre d'absorption X de Skinner et Johnston. 
de confirmer la validité des règles de transition optique par ce type d'excitation. C'est-à-dire que si $l$ désigne le nombre quantique azimutal, les transitions seront telles que $\Delta l= \pm 1$. Le spectre $\mathrm{K}$ du lithium (Fig. 10) obtenu par Colliex et Jouffrey [22] présente les mêmes
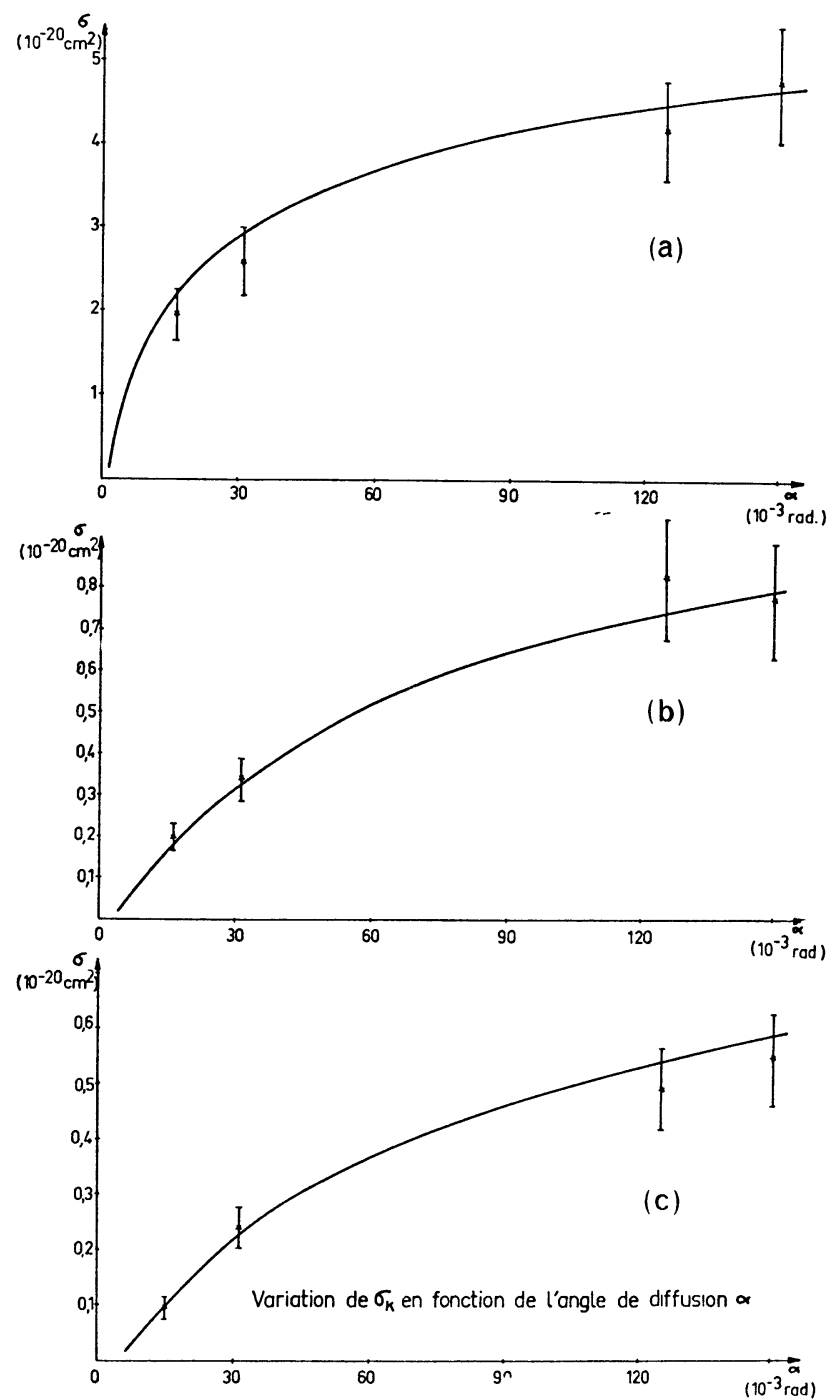

FIG. 10. - Variation de la section efficace de diffusion correspondant à l'excitation du niveau $K$ par des électrons d'énergie $E_{0}=60 \mathrm{keV}$ en fonction de l'angle maximal de diffusion $\alpha$. a) Dans le carbone. b) Dans le magnésium. c) Dans l'alumiminium.

maxima que le spectre d'absorption $\mathrm{X}$ dû à Skinner et Johnston [23]. La même analogie peut être notée entre le spectre relatif à la transition $\mathrm{L}_{23}$ de l'aluminium observé par ces premiers auteurs et le spectre correspondant d'absorption X dû à Sagawa [24].

La similitude des spectres $\mathrm{K}$ de l'aluminium, du magnésium et du silicium qui ont été obtenus au laboratoire [8] et figure 2, correspond bien au fait que ces éléments ont le même type de structure électronique. La pente de la distribution après le seuil $\mathrm{K}$ est importante, à la différence de ce qui est observé pour la transition $\mathrm{L}_{23}$. Le caractère essentiel du profil correspondant à celle-ci est l'apparition d'un maximum pour une énergie bien supérieure (de plusieurs dizaines d'eV) au niveau de Fermi, en accord avec l'existence d'une bande à caractère $d$ très marqué autour d'une énergie de quelques dizaines d'eV au-delà de l'énergie de Fermi. Le profil qui est associé à la transition $\mathrm{K}$ dans ces éléments semble confirmer le caractère $\mathrm{p}$ de la bande située juste au-dessus du niveau de Fermi.

Les spectres montrent cependant que la méthode ne permet pas à l'heure actuelle d'analyser les structures fines au voisinage des seuils d'absorption. La structure observée ne peut être reliée à la courbe des densités d'état des énergies des électrons dans le solide que de manière très imparfaite. Des expériences plus précises de Trebbia et Colliex [25, 26] sur l'excitation des niveaux atomiques caractéristiques dans les métaux de transition et les éléments des terres rares, soulignent ce résultat. Cependant ces auteurs ont pu associer dans le spectre d'Ytterbium, des modifications de structure des spectres, à des changements de phases liés à la formation de cristaux d' $\mathrm{Yb}_{2} \mathrm{O}_{3}$ par oxydation. Ces derniers peuvent être observés par microscopie électronique. Ce résultat montre que la technique peut avoir une application dans l'étude des alliages. Mais c'est surtout la détermination des seuils dus à l'excitation des niveaux les plus profonds qui a été utilisée pour l'analyse chimique locale.

\subsection{Probabilité D'excitation des niveaux ato-} MIQUES. - Les libres parcours moyens correspondant à l'excitation du niveau $\mathrm{K}$ ont été déterminés à $60 \mathrm{kV}$ dans le cas du carbone, du magnésium et de l'aluminium [8], et entre 300 et $1200 \mathrm{kV}$ [27] dans celui du carbone et de l'aluminium. Dans les deux tableaux suivants, les valeurs expérimentales de $\lambda_{\mathrm{K}}$ sont comparées aux valeurs $\lambda_{\mathrm{KB}}$ calculées en utilisant les résultats du traitement de Bethe [28] et au-delà de $300 \mathrm{kV}$ à l'expression de $\lambda_{\mathrm{KF}}$ qui peut être déduite du traitement de Fano dans le cas relativiste [29].

$$
\lambda_{\mathrm{KB}}=\frac{1}{n_{\mathrm{a}} \cdot \sigma_{\mathrm{KB}}}
$$

avec

$$
\begin{gathered}
\sigma_{\mathrm{KB}}=\frac{f_{\mathrm{K}}}{E_{\mathrm{K}} \cdot \beta^{2}} \log \frac{\alpha^{2}+\theta_{\mathrm{E}}^{2}}{\theta_{\mathrm{E}}^{2}} \\
\lambda_{\mathrm{KF}}=\frac{1}{n_{\mathrm{a}} \cdot \sigma_{\mathrm{KF}}}
\end{gathered}
$$

avec

$$
\begin{array}{r}
\sigma_{\mathrm{KF}}=\sigma_{\mathrm{KB}}+\frac{f_{\mathrm{K}}}{E_{\mathrm{K}} \cdot \beta^{2}}\left[\log \frac{1}{1-\beta^{2}}-\beta^{2}+\frac{\beta^{2} \theta_{\mathrm{E}}^{2}\left(1-\beta^{2}\right)}{\alpha^{2}+\theta_{\mathrm{E}}^{2}\left(1-\beta^{2}\right)}\right. \\
\left.-\log \frac{\alpha^{2}+\theta_{\mathrm{E}}^{2}}{\alpha^{2}+\theta_{\mathrm{E}}^{2}\left(1-\beta^{2}\right)}\right]
\end{array}
$$

où $n_{\mathrm{a}}$ est le nombre d'atomes par unité de volume, $\sigma_{\mathbf{K}}$ la section efficace de diffusion, $f_{\mathbf{K}}$ et $E_{\mathbf{K}}$ la force 
d'oscillateur et la perte d'énergie correspondant à l'excitation du niveau $\mathrm{K}, \alpha$ est l'angle maximal de diffusion et

$\theta_{\mathrm{E}}=\frac{E_{\mathrm{K}}\left(E_{0}+1\right)}{E_{0}\left(E_{0}+2\right)}$.
$\alpha=150 \mathrm{mrad}$

$$
\alpha=75 \mathrm{mrad}
$$$$
\alpha=10 \mathrm{mrad}
$$

$E_{0} \mathrm{KeV}$
-
300
500
750
1000
1200

$$
\lambda_{\mathrm{KB}} \quad \lambda_{\mathrm{KF}} \quad \lambda_{\mathrm{K}}^{\exp }
$$$$
\lambda_{\mathrm{K}}
$$$$
\lambda_{\mathrm{K}}
$$

$\begin{array}{llr}\overline{6,4} & \overline{6,2} & 10,8 \\ 7,6 & 6,8 & 6,2 \\ 7,6 & 7 & 9,2 \\ 7,8 & 7 & 9,5 \\ 7,8 & 6,9 & 7,6\end{array}$

$$
11
$$$$
11 \quad 10,4
$$$$
11,8 \quad 10,8
$$$$
1210,6
$$$$
11,9 \quad 10,2
$$$$
11,8 \quad 9,9
$$

L'imprécision sur les valeurs expérimentales $\lambda_{\mathrm{K}}^{\text {exp }}$ est inférieure à $30 \%$ à très haute tension et à $15 \%$ à plus basse tension. On peut considérer que les résultats théoriques et expérimentaux sont en bon accord. A faible énergie, Egerton [30] et Isaacson [31] ont obtenu des résultats comparables dans le cas du carbone. Il semble donc, qu'au moins pour les éléments de faible numéro atomique, le traitement théorique de Bethe rende bien compte de la diffusion inélastique d'une particule chargée par un atome.

Ces résultats montrent que le libre parcours moyen relatif à l'excitation de niveaux atomiques profonds augmente avec l'énergie des électrons incidents. Il tend vers une valeur maximale aux environs de $1 \mathrm{MeV}$, puis décroît.

Une étude plus particulière de la variation de la section efficace de diffusion montre sur la figure 9, sa variation en fonction de l'angle maximal de diffusion $\alpha$ [8]. Les électrons diffusés par excitation du niveau atomique profond $\mathrm{K}$ se répartissent principalement dans un petit angle, inférieur à $10^{-1}$ radian pour le carbone et à une valeur un peu plus grande pour les éléments de numéro atomique plus élevé [31].

Les valeurs de $\lambda_{\mathrm{K}}$ sont 50 à 100 fois supérieures aux valeurs associées à la diffusion par plasmon dans le cas du carbone, et de 300 à 1500 fois dans le cas du magnésium et de l'aluminium. Ceci montre bien la faiblesse des probabilités d'excitation des niveaux profonds qui interviennent peu dans la formation des images en microscopie électronique.

4.3 Application a L'ANAlyse ChimiQue. - En dépit de leur faible intensité, les signaux dus à l'excitation des niveaux atomiques profonds ont permis de parvenir à une analyse chimique dans de très petites portions de l'objet comme l'ont montré d'abord Wittry, Ferrier et Cosslett [33], puis Colliex et Jouffrey [34]. Cette méthode s'applique essentiellement à des éléments de faible numéro atomique (seule l'excitation d'un électron d'un niveau donné intervient ici).

D'après les éq. (2), la probabilité d'excitation est inversement proportionnelle à $E_{\mathrm{K}^{\prime}}$ donc au numéro atomique. La distribution angulaire des électrons ayant excité le niveau profond est proportionnelle au facteur $\left(\alpha^{2}+\theta_{\mathrm{E}}^{2}\right)^{-1}$. Bien que l'ouverture des spectromètres soit limitée, ceux-ci collectent la majeure partie des électrons diffusés à la suite de l'excitation du niveau profond, d'autant plus que $Z$ est plus petit et que l'énergie $E_{0}$ est plus grande. Ce dernier point ajouté au fait que le fond continu d'un spectre de pertes d'énergie décroît lorsque la tension accélératrice s'élève peut expliquer que des excitations de niveaux atomiques plus profonds aient été mises en évidence à très haute tension [21].

Les inconvénients majeurs de cette méthode paraissent être liés à la faiblesse du rapport de l'intensité du signal utile sur le fond continu. Ce rapport est amélioré en utilisant de très petits angles de diffusion comme l'a souligné Egerton [35]. Dans tous les cas, il est préférable d'utiliser les pertes liées à l'excitation des niveaux les plus profonds $\mathrm{K}$ ou $\mathrm{L}$ dont les énergies sont mieux séparées.

Comme dans le cas des plasmons, deux méthodes ont été utilisées pour appliquer la détection des pertes liées à l'excitation des niveaux atomiques à l'analyse chimique. La première consiste à sélectionner une portion de l'image de l'objet à étudier et à former le spectre des pertes d'énergie des électrons dans cette portion de l'image. Dans la deuxième, l'image est filtrée dans son ensemble en sélectionnant les électrons qui correspondent à une perte caractéristique donnée.

La première méthode a permis de mettre en évidence la présence de bore en proportion de $0,1 \%$ dans une matrice de silicium. Elle a été largement appliquée à l'étude de grains de poussières lunaires et de feldspaths qui ne peuvent pas être étudiés à basse tension à cause de leur épaisseur, de l'ordre de $1 \mu \mathrm{m}$ [36]. Nous donnons trois exemples de ces études.

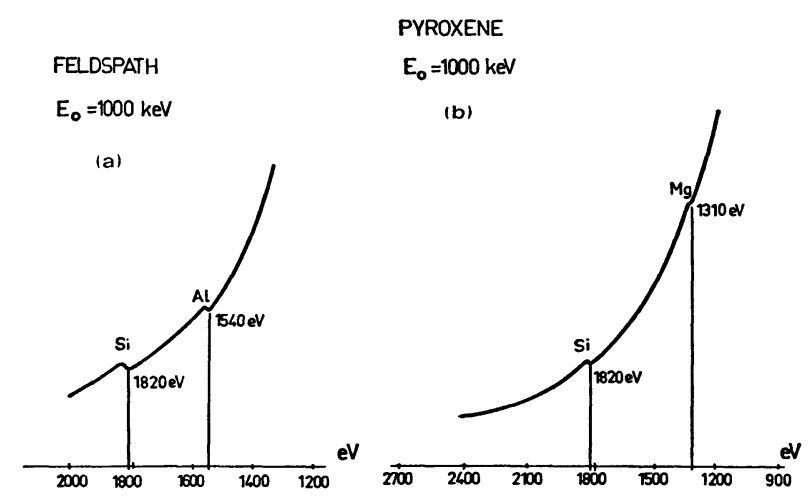

FIG. 11. - Spectres de pertes d'énergie. a) Caractérisation d'un grain de feldspath. b) Caractérisation d'un grain de pyroxène. 


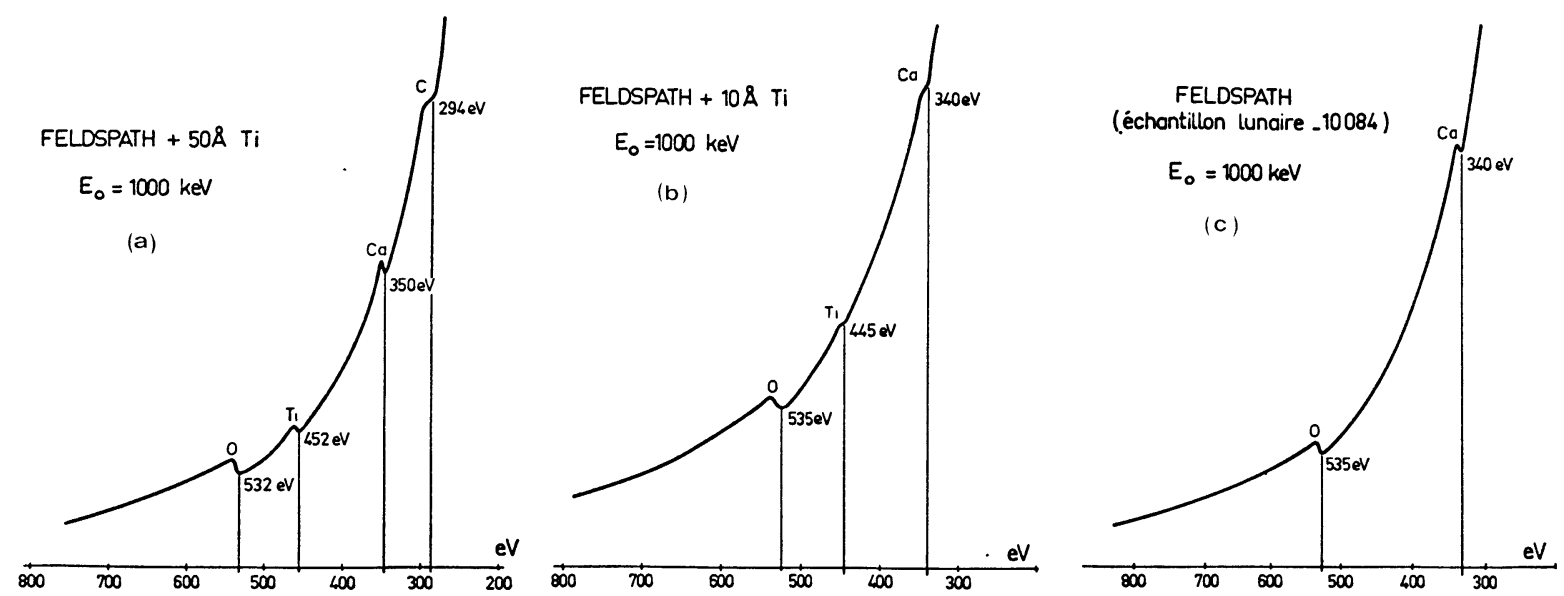

FIG. 12. - Caractérisation du titane sur des grains de feldspath de diverses origines. Spectres de pertes d'énergie d'électrons de $1 \mathrm{MeV}$ dans : a) Grain de feldspath recouvert d'une évaporation de titane d'épaisseur $50 \AA . b$ ) Grain de feldspath recouvert

d'une évaporation de titane d'épaisseur $10 \AA . c$ ) Grain de feldspath d'origine lunaire recouvert d'une peau amorphe.

4.3.1 Les grains isolants rencontrés dans les poussières lunaires sont soit des feldspaths $(\mathrm{Al}, \mathrm{Si}, \mathrm{Ca})$, soit des pyroxènes $(\mathrm{Si}, \mathrm{Mg}, \mathrm{Fe}, \mathrm{Ca})$. L'analyse spectroscopique permet de mettre en évidence les distributions correspondant à l'excitation des niveaux $\mathrm{K}$ des divers éléments. Les courbes de la figure 11 montrent celles qui sont relatives à l'aluminium à $1540 \mathrm{eV}$ et au silicium à $1820 \mathrm{eV}$ (Fig. 10a) pour le feldspath et au magnésium à $1310 \mathrm{eV}$ et au silicium à $1820 \mathrm{eV}$ (Fig. 10b) pour le pyroxène.

Dans cette expérience, le spectre des pertes d'énergie permet de reconnaître la nature de chacun des grains qui sont difficiles à identifier sur les images de microscopie.

4.3.2 Après un calibrage préalable à l'aide d'évaporations de titane d'épaisseur connue de 10 à $250 \AA \AA$ sur des feldspaths, nous avons recherché la présence de titane dans la peau amorphe qui recouvre certains grains d'origine lunaire. Sur la figure 12, nous comparons l'enregistrement microdensitométrique de la portion du spectre comprise entre 200 et $700 \mathrm{eV}$.

La figure $12 a$ correspond à une épaisseur de $50 \AA \AA$ de titane. Les seuils relatifs aux excitations des niveaux $\mathrm{K}$ du carbone, $\mathrm{L}_{23}$ du calcium, $\mathrm{L}_{23}$ du titane et $\mathrm{K}$ de l'oxygène sont observés successivement.

Sur la figure $12 b$, pour une épaisseur de titane de $10 \AA$, la distribution correspondant à la transition $\mathrm{L}_{23}$ du titane est beaucoup moins intense. Par contre, elle n'apparaît plus sur la figure $12 c$ obtenue avec un grain de feldspath d'origine lunaire recouvert d'une peau amorphe de $500 \AA \AA$ d'épaisseur. Cette analyse permet de conclure que la teneur en titane de la peau amorphe est très faible. Elle ne confirme pas l'hypothèse qui a été avancée que ce dépôt était dû à des vapeurs métalliques.

4.3.3 La figure 13 montre les raies correspondant aux divers constituants de l'ilménite $\left(\mathrm{FeTiO}_{3}\right)$, le titane, l'oxygène et le fer. Le titane et le fer sont ici mis

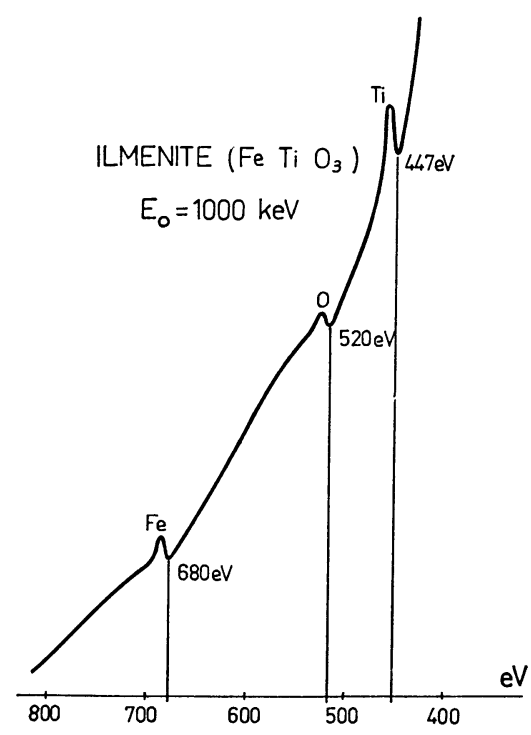

FIG. 13. - Spectre de pertes d'énergie d'électrons de $1 \mathrm{MeV}$ au travers d'un échantillon d'ilménite, zone des pertes d'énergie $<1000 \mathrm{eV}$

en évidence par la distribution correspondant à l'excitation des niveaux $\mathrm{L}_{23}$.

La sensibilité de cette technique de spectrométrie électronique d'aire sélectionnée paraît être à l'heure actuelle de l'ordre de $0,1 \%$. Elle a été appliquée à des volumes de l'ordre de $1 \mu^{3}$. Elle pourra être améliorée en utilisant des détecteurs d'électrons plus sensibles et plus précis que les plaques photographiques. L'adaptation d'un analyseur d'énergie sur le microscope $3 \mathrm{MV}$ doit permettre d'accroître la zone des pertes d'énergie utiles et donc le nombre des éléments qui peuvent être détectés [37].

Les résultats acquis avec la deuxième méthode basée sur le filtrage de l'image, ont été obtenus dans des microscopes disposant d'un système de filtrage du type Castaing-Henry. Ainsi Colliex et Jouffrey [34] ont comparé les images d'un cristal de graphite obtenues 
en ne conservant que les électrons qui ont été diffusés élastiquement, avec une perte par plasmon, puis une perte inférieure, égale ou supérieure à la perte par excitation du niveau $\mathrm{K}$ du carbone située à $285 \mathrm{eV}$ (Fig. 14).
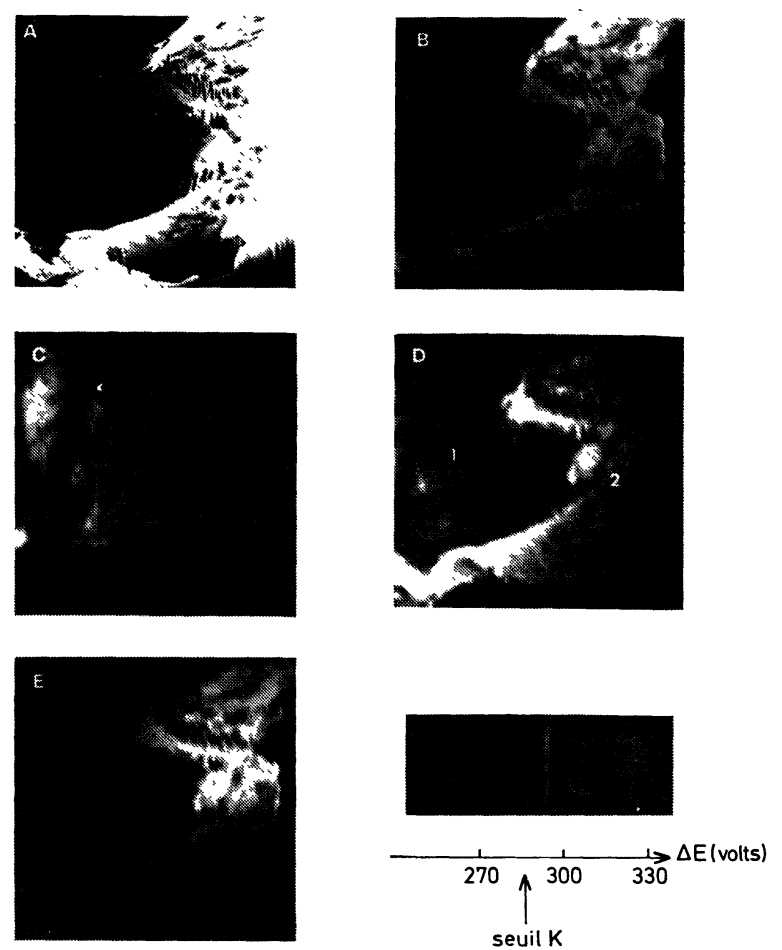

FIG. 14. - Image d'un cristal de graphite dans diverses conditions de filtrage $E_{0}:$ A) Image élastique. B) Image formée avec des électrons ayant excité un plasmon. C) Image formée avec des électrons dont la perte d'énergie se situe juste avant le seuil d'excitation K. D) Image formée avec des électrons ayant subi une perte d'énergie caractéristique du niveau K. E) Image formée avec des électrons ayant perdu une énergie correspondant à la fin de la bande K. F) Spectre des pertes d'énergie au voisinage du seuil $K$.

Des expériences analogues ont été faites par Egerton et Whelan [37] sur des particules de $\mathrm{BN}$ et de $\mathrm{MgO}$. Celles sur le $\mathrm{MgO}$ ont été reprises au laboratoire à $60 \mathrm{kV}$ [8]. Dans cette méthode la difficulté essentielle vient de ce que les électrons ayant subi des pertes caractéristiques se superposent à ceux appartenant au fond continu. Les effets respectifs des deux types de pertes sont difficiles à séparer sur des images filtrées. Ils peuvent donner lieu à des modifications identiques du contraste.

Il semblerait que les techniques utilisant un microscope à balayage par transmission, puissent permettre de résoudre ce problème. La contribution due au fond continu peut être éliminée en soustrayant de l'image les électrons situés juste avant le seuil $\mathrm{K}$. Il suffit pour cela de soustraire les signaux recueillis par l'intermédiaire de deux fentes séparées par quelques dizaines d'eV et disposées dans le plan du spectre. Par ailleurs, cette technique peut se prêter à l'application du traitement numérique des spectres. A la condition de disposer de sources d'électrons suffisamment intenses et de détecteurs d'électrons assez sensibles, la résolution spatiale de l'analyse peut être très proche de celle du microscope électronique et donc de l'ordre du nanomètre.

5. Autres méthodes d'analyse en microscopie électronique. - Plusieurs méthodes ont été récemment développées pour mener à bien une analyse chimique à petite échelle dans le cas des couches minces. Elles ont pour origine l'analyse $\mathrm{X}$ proposée par $\mathrm{R}$. Castaing [39]. Elles se sont beaucoup développées autour du microscope électronique à balayage, au point de donner lieu à la commercialisation de dispositifs d'analyse.

Il faut distinguer deux types de méthodes d'analyse $\mathrm{X}$, les méthodes dispersives et les méthodes non dispersives. Dans le premier cas, la résolution des spectromètres est de l'ordre de $10 \mathrm{eV}$. Le deuxième type de méthode est le plus facile à mettre en œuvre mais la résolution se situe actuellement aux environs de $150 \mathrm{eV}$. A cause de la diffusion des électrons dans l'échantillon, la résolution spatiale de ces méthodes présente une limitation inférieure. Le diamètre de la source des rayons $\mathrm{X}$ analysés augmente avec la profondeur de la zone considérée dans l'échantillon. Dans des échantillons massifs, pour des électrons incidents d'énergie $E_{0} \sim 30 \mathrm{keV}$, la résolution spatiale de l'analyse est limitée à une valeur comprise entre 1000 et $5000 \AA$. Naturellement dans les échantillons minces, de quelques centaines d'angströms, cette résolution peut être abaissée mais il paraît peu probable qu'elle puisse être rendue inférieure à $200 \AA$ [40]. Ces méthodes sont mal adaptées à l'analyse des éléments de faible numéro atomique. En effet, le nombre de quanta $X$ émis par excitation d'une couche atomique déterminée décroît rapidement avec le numéro atomique. Lors de l'ionisation de la couche $\mathrm{K}$, du sodium, il faut produire 40 excitations en moyenne pour qu'un photon $\mathrm{X}$ soit émis ; dans le cas de la couche $\mathrm{K}$ du carbone ce nombre passe à 400 [41]. La situation est encore plus défavorable pour l'excitation de niveaux atomiques moins profonds L ou M. Par ailleurs, l'efficacité de collection des photons $X$ est très faible, inférieure à $10^{-2}$ [42]. En effet, les photons $X$ sont émis uniformément dans un angle solide de $4 \Pi$ steradians alors que les détecteurs ont une ouverture utile très limitée.

Ces deux points constituent la raison essentielle pour laquelle les méthodes d'analyse $X$ sont peu efficaces pour la détection des éléments de très faibles numéros atomiques tels que l'oxygène, le carbone, le bore, etc...

Cette lacune peut être en partie comblée par la spectrométrie des électrons Auger. Dans ce cas en effet, quel que soit l'élément, le vide laissé par l'ionisation d'un électron $\mathrm{K}$ peut être rempli de différentes manières et le signal à analyser est relativement important. Cependant, à cause de la faible énergie des électrons Auger, la technique n'apporte des résultats que dans le 
cas d'une analyse de surface, sur des épaisseurs de quelques dizaines d'angströms seulement.

6. Analyse quantitative. - Les techniques de spectrométrie $X$ ont pu évoluer vers l'analyse quantitative. Dans ce domaine, elles se heurtent souvent à un certain nombre de difficultés qui sont d'ailleurs communes à toutes les techniques d'analyse utilisant le microscope électronique, qu'elles soient basées sur l'analyse X, sur la spectrométrie Auger ou sur l'analyse des pertes d'énergie des électrons transmis.

Dans le cas des matériaux cristallins, conformément aux résultats de la théorie dynamique, il apparaît à l'intérieur du cristal plusieurs ondes $\Psi^{(i)}$ définies par des fonctions de Bloch. Chacune de ces ondes représente la somme d'une onde transmise et d'ondes diffractées. Les ondes ne sont pas transmises de la même manière dans le cristal. La probabilité de présence des électrons au niveau des plans atomiques change d'une onde à l'autre. Dans le cas de la théorie à deux ondes, elle est maximum pour l'une et minimum pour l'autre. Cet effet de canalisation au niveau des plans atomiques est gênant pour l'analyse : l'onde centrée sur les noyaux étant plus efficace et les poids des diverses ondes de Bloch variant avec l'angle d'incidence sur le cristal [43, 44]. Cet effet est encore mal connu et il semble que le comportement des diverses ondes dans la production du rayonnement de freinage n'ait pas encore été analysé.

Dans le cas d'objets amorphes les problèmes d'analyse quantitative se heurtent d'abord au problème de la détermination de l'épaisseur de l'échantillon étudié. Pour cette raison, Hall et Werba [45] ont essayé de développer une méthode dans laquelle le résultat est indépendant de ce paramètre. Le rapport $R$ du nombre de photons $n_{\mathrm{x}}$ correspondant à la raie caractéristique observée et du nombre de photons $n_{\beta}$ émis simultanément et appartenant au fond continu est proportionnel au rapport $\frac{N_{\mathrm{x}}}{\sum N Z^{2}}$

$$
R=\frac{n_{\mathrm{x}}}{n_{\beta}} \simeq \frac{N_{\mathrm{x}}}{N Z^{2}}
$$

$N_{\mathrm{x}}$ est le nombre des atomes de l'élément étudié dans le volume considéré et $\Sigma N Z^{2}$ représente, comme l'a montré Kramers [46], la part due au Bremstrahlung. La validité de la méthode dépend essentiellement des conditions dans lesquelles la formule de Kramers est vérifiée, c'est-à-dire d'après Hall et Werba [45] pour $8<Z<74$. Dans les expériences [47] les épaisseurs étudiées n'excédaient pas $4000 \AA$. Par ailleurs, il semble indispensable que l'épaisseur de la couche soit uniforme et que le poids atomique moyen de l'échantillon soit à peu près constant.

Duncumb [48] a proposé une technique qui utilise comme référence l'émission d'échantillons massifs étalons. Elle a été reprise par Jacobs et Baborovska [49]. La technique de Cliff et Lorimer [50] procède en deux étapes. Le faisceau d'électrons est d'abord focalisé sur une petite surface puis défocalisé. Il éclaire alors une région plus étendue, représentative de la composition moyenne de la matrice. Le rapport des intensités $X, I_{1}$ et $I_{2}$, recueillies dans les deux cas est proportionnel au rapport des compositions moyennes $C_{1}$ et $C_{2}$ du matériau.

$$
\frac{I_{1}}{I_{2}}=K \frac{C_{1}}{C_{2}}
$$

$K$ est une constante liée aux conditions expérimentales. Les expériences ont montré que cette technique peut être utilisée pour l'analyse quantitative jusqu'aux épaisseurs maximales de pénétration utile des électrons au sens de la microscopie électronique à transmission, c'est-à-dire, jusqu'à une épaisseur de quelques milliers d'angströms.

Ces quelques exemples montrent que les méthodes d'analyse quantitative sont en cours de développement. Dans les méthodes de spectrométrie des pertes d'énergie des électrons, aucun résultat purement quantitatif n'a encore été obtenu. Cette phase de l'analyse pourra être envisagée avec l'emploi de méthodes de détection et de traitement du signal analogues à celles qui sont adoptées pour la spectrométrie $\mathrm{X}$.

Les résultats récents de plusieurs groupes de chercheurs, par exemple Colliex C. [7], Egerton R. F. [30], Isaacson M. [31], et ceux qui sont obtenus à Toulouse nous conduisent à la conclusion que l'analyse locale, basée sur la spectrométrie des pertes d'énergie des électrons dues à l'excitation des niveaux atomiques profonds dans une aire sélectionnée de l'objet, pourra connaître un développement intéressant. Les résultats sont encore fragmentaires. Les perfectionnements des microscopes électroniques, le choix de nouveaux dispositifs d'analyse des pertes d'énergie et de filtrage, ainsi que de nouvelles méthodes de détection des électrons, en liaison avec les possibilités d'analyse numérique, doivent permettre d'exploiter complètement cette technique. Elle peut alors présenter de nombreux avantages pour l'analyse des éléments de faible numéro atomique et dans le domaine de la résolution spatiale.

\section{Bibliographie}

[1] Ruthemann, G., Naturwissenschaften 29 (1941) 648.

[2] Hillier, J., Phys. Rev. 64 (1943) 318.

Hillier, J. et BaKer, R. F., J. Appl. Phys. 15 (1944) 663.

[3] Castaing, R. et Henry, L., C. R. Hebd. Séan. Acad. Sci. 255 (1962) 76.

HenRy, L., Thèse Paris (1964).
[4] Watanabe, H., J. Phys. Soc. Japan 9 (1954) 920.

[5] Klemperer, O., Rep. Prog. Phys. 28 (1965) 77.

[6] Metherell, A. J. F., Adv. Opt. Electron Micros. 4 (1971) 263.

[7] Colliex, C, Analytical Electron Microscopy Kontron Seminar (Münich) 1975. 
[8] KinN, Y., Thèse spécialité, Toulouse (1975).

[9] Sevely, J., Perez, J. Ph. et Jouffrey, B., C. R. Hebd. Séan. Acad. Sci. 276 (1973) 515.

[10] Вонм, D., Pines, D., Phys. Rev. 82 (1951) 625.

Nozières, D., Pines, D., Phys. Rev. 9 (1958) 470.

Pines, D., The Many Body Problem (Benjamin, New York) 1961.

[11] Raether, H., Springer Tracts Mod. Phys. 38 (1965) 84.

[12] Sevely, J., Perez, J. Ph., Kinn, Y. and Jouffrey, B., V. U. V. Radiation Physics, Hamburg (Pergamon) 1974, p. 618.

[13] Burge, R. E. et Misell, D. L., Phil. Mag. 18 (1968) 261.

[14] Ashley, J. C. and Ritchie, R. H. Phys. Stat. Sol. 40 (1970) 623.

[15] Pines, D., Phys. Rev. 92 (1953) 626.

[16] Cundy, S. U., Metherell, A. J. F., Whelan, M. J., J. Sci. Instrum. 43 (1966) 712.

[17] Doig, P., Edington, J. W. et Jacobs, M. H., Phil. Mag. 31 (1975) 2.

[18] Spalding, D. R., Villagrana, R. E. and Chadwick, G. A., Phil. Mag. 20 (1969) 471.

[19] Castaing, R., El Hili, A. et Henry, L., C. R. Hebd. Séan. Acad. Sci. 261 (1965) 3399.

[20] Henoc, P. and Henry, L., J. Physique Colloq. 31 (1970), C 4-55.

[21] Sevely, J., Perez, J. Ph. and Jouffrey, B., High Voltage Electron microscopy, (Academic Press, Oxford) 32 (1974).

[22] Colliex, C. et Jouffrey, B., Phil. Mag. 25 (1972) 471. Colliex, C., Thèse Orsay (1970).

[23] Skinner, H. W. B. et Johnston, J. E., Proc. R. Soc. A, 161 (1937) 420.

[24] Sagawa, T. et coll., J. Phys. Soc. Japan 21 (1966) 2602.

[25] TrebBiA, P., Thèse spécialité, Orsay, (1973).

[26] Trebbia, P. and Colliex, C., 8th Int. Cong. of Electron Microscopy, Canberra, I, (1974), 382.

[27] Perez, J. Ph., Sevely, J. and Jouffrey, B., 8th Int. Cong. of Electron Microscopy, Canberra, I, (1974), 384.

[28] Bethe, H., Ann. Phys. 5 (1930) 325.
[29] Fano, U., Phys. Rev. 102 (1956) 385.

[30] Egerton, R. F., Phil. Mag. 31 (1975) 199.

[31] Isaacson, M., J. Chem. Phys. 56 (1972) 1813.

[32] IsaAcson, M. and Johnson, P., Scanning Electron Microscopy, I, Chicago, IITRI, (1975), 157.

[33] Wittry, D. B., Ferrier, R. P. and Cosslett, V. E., Brit. 5. Appl. Phys. 2 (1969) 1767.

[34] Colliex, C. and Joufrrey, B., C. R. Hebd. Séan. Acad. Sci. 720 (1970) 144 et 673.

[35] Egerton, R. F., Proc. Joint. Meeting, London, (1974).

[36] Sevely, J., Perez, J. Ph., Jouffrey, B., Bibring, J. P. and Maurette, M., $4^{\mathrm{e}}$ Congrès International de M. E. H. T., Toulouse (1975).

[37] Perez, J. Ph., Zanchi, G., Sevely, J. and Jouffrey, B., Optik, à paraître.

[38] Egerton, R. F. and Whelan, M. J., 8th Intern. Congress on Electron Microscopy, Canberra 1, (1974), 384.

[39] Castaing, R., Thèse Université de Paris (1951), publication O. N. E. R. A. $n^{\circ} 55$.

[40] Woolf, R. J., and Joy, D. C., Proc 5th Eur. Cong. Electron Microscopy, (1972), 144.

[41] BambynCK, W. et col., Rev. Mod. Phys. 44 (1972) 714.

[42] Hall, T. A., Physical Techniques in Biological Research I A, 2nd ed., (Academic Press, N. Y.) 1971.

[43] Duncumb, P., Phil. Mag. 7 (1962), 2101 ; J. Sci. Instrum. 2 (1969) 553.

[44] Castaing, R., Henoc, P., Henry, L. et Natta, M., $C$. $R$. Hebd. Séan. Acad. Sci. 265, (1967) 1293.

[45] Hall, T. A. et Werba, P. R., Proc. 25th ann. meeting of E. M. A. G., (1971), 146.

[46] Kramers, H. A., Phil. Mag. 46 (1923) 836.

[47] Hall, T. A., Clarke Anderson, H. and Appleton, T., $J$. Micros. 99 (1973) 177.

[48] Duncumb, P., J. Micros. 7 (1968) 581.

[49] Jacobs, M. H. et Baborovska, J. Proc. 5th Eur. Cong. Electron Microscopy, (1972), 136.

[50] Cliff, G. et LoRimer, G. W., Proc. 5th Eur. Long. Electron Microscopy (1972), 140. 